\title{
Separation of a Signal of Interest from a Seasonal Effect in Geophysical Data: I. EI Niño/La Niña Phenomenon
}

\author{
David H. Douglass \\ Department of Physics and Astronomy, University of Rochester, Rochester, USA \\ E-mail:douglass@pas.rochester.edu \\ Received April 12, 2011; revised July 9, 2011; accepted September 2, 2011
}

\begin{abstract}
Geophysical signals $N$ of interest are often contained in a parent signal $G$ that also contains a seasonal signal $X$ at a known frequency $f_{X}$. The general issues associated with identifying $N$ and $X$ and their separation from $G$ are considered for the case where $G$ is the Pacific sea surface temperature monthly data, SST3.4; $N$ is the El Niño/La Niña phenomenon and the seasonal signal $X$ is at a frequency of $1 /(12$ months). It is shown that the commonly used climatology method of subtracting the average seasonal values of SST3.4 to produce the widely used anomaly index Nino3.4 is shown not to remove the seasonal signal. Furthermore, it is shown that the climatology method will always fail. An alternative method is presented in which a $1 / f_{X}(=12$ months $)$ moving average filter $\mathcal{F}$ is applied to SST3.4 to generate an El Niño/La Niña index $N_{\mathrm{L}}$ that does not contain a seasonal signal. Comparison of $N_{\mathrm{L}}$ and Nino3.4 shows, among other things, that estimates of the relative magnitudes of El Niños from index $N_{\mathrm{L}}$ agree with observations but estimates from index Nino3.4 do not. These results are applicable to other geophysical measurements.
\end{abstract}

Keywords: El Niño/La Niña, Climate, Seasonal

\section{Introduction}

The study of many geophysical phenomena starts with a parent signal $G$ that contains a signal of interest $N$ plus a seasonal signal $X$ at frequency $f_{X}$ and its harmonics. The first task of many studies is to separate $X$ from $G$ to obtain $N$. If the spectral content of $N$ lies mostly at frequencies below $f_{X}$, then separation may straightforwardly be achieved: the Fourier transform of $G$ is taken; the part of the spectrum at $f_{X}$ and higher frequencies is removed and the transform back to the time domain yields $N$. Douglass [1] has pointed out that the separation can easily be obtained in the time domain by applying a $1 / f_{X}$ moving average filter $\mathcal{F}$ to $G$. Specifically, application of $\mathcal{F}$ to $G$ yields a low frequency (lower than $f_{X}$ ) signal $N_{\mathrm{L}}$ containing the signal of interest. This paper shows, additionally, that subtraction of $N_{\mathrm{L}}$ from $G$ produces a high frequency ( $f_{X}$ and higher) signal $N_{\mathrm{H}}$ that contains the seasonal signal. These methods are demonstrated for the case where $G$ is the equatorial Pacific sea surface temperature (SST), $N$ is an index describing the El Niño/La Niña phenomenon, and the seasonal frequency $f_{X}$ is $1 /(12$ months) which requires that $\mathcal{F}$ be a 12 month moving average filter.
Moving average filters applied to SST data have been used by Federov and Philander [2] (FP) to study the El Niño/La Niña phenomenon. They applied a 9-month moving average filter to SST data. An El Niño/La Niña anomaly signal was obtained by subtracting the average SST from the filtered signal. This anomaly was compared to one where the subtraction was a 10 -year moving average. The differences were considerable. They stated: “... the episodes [El Niños] of 1982 and 1997 appear less exceptional..." It is noted that the FP anomalies differ from those calculated with a 12-month filter and those calculated from the climatology method (next paragraph).

A widely used different scheme is the "climatology" method that purports to remove the seasonal signal. For monthly data the climatology $(C)$ is a set of 12 numbers -one for each month where the value for each month is an average over $G$ for a fixed period (usually 30 years).

An index $N$ is defined which is the value of $G$ for each month minus the corresponding value of $C$. This method is applied to the temperature data, SST3.4, from Pacific SST Region 3.4 to create the commonly used anomaly index Nino3.4 [3]. It is implied that Nino3.4 is "seasonal free". However, Douglass [1] showed that the Fourier spectrum of Nino3.4 had a substantial spectral compo- 
nent at a frequency of $1 /$ year.

In Section 2, data sources and the filter F are described. The new indices are also defined. In Section 3 various indices are calculated and discussed. The Lyapunov exponents of various time series are also determined. Section 4 contains a discussion. Section 5 has a summary.

\section{Data Source and Methods}

All data are monthly time series.

\subsection{Source}

In a general study with the objective of finding the location in the tropical Pacific with the strongest correlation with the core El Nino Southern Oscillation (ENSO) phenomenon Barnston, Chelliah and Goldenberg [4] (BCG) defined a sea surface temperature (SST) Region 3.4 (latitude: $5 \mathrm{~S}$ to $5 \mathrm{~N}$; longitude: $120 \mathrm{~W}-170 \mathrm{~W}$ ) that overlaps previously defined Region 3 and Region 4 . The average SST of Region3.4 is named SST3.4 and ranges between $24^{\circ} \mathrm{C}$ and $30^{\circ} \mathrm{C}$. BCG defined a corres- ponding index of anomalies, Nino3.4, which is SST3.4 with the seasonal signal nominally removed by the climatology method (next section). The monthly values of SST3.4 and Nino3.4 are given by the Climate Prediction Center [3]. The values are from January 1950 to the present (February 2011). Figure 1(a) shows SST3.4 and Nino3.4 is shown in Figure 2(a). The familiar El Niños of 19831984 and 1997-1998 are indicated.

\subsection{Climatology Method}

This is a scheme that purports to remove the seasonal effect from geophysical data. For the monthly SST3.4 data the climatology $(C)$ is a set of 12 numbers - one for each month where the value for each month is an average over SST3.4 for a fixed period (usually 30 years). The amplitude of the periodic function varies from January to December and is the same for each year. The second part of the climatology method applied to SST3.4 is to define an anomaly index Nino3.4 that is the value of SST3.4 for each month minus the corresponding value of $C$. It is noted for later discussion that mathematically $C$ is a function consisting of a constant plus a periodic term (period $=1$ year) with monthly amplitudes that are the same for each year.

\subsection{The 12-Month Digital Filter $\mathcal{F}$.}

Consider monthly time-series data that have been put through the digital filter

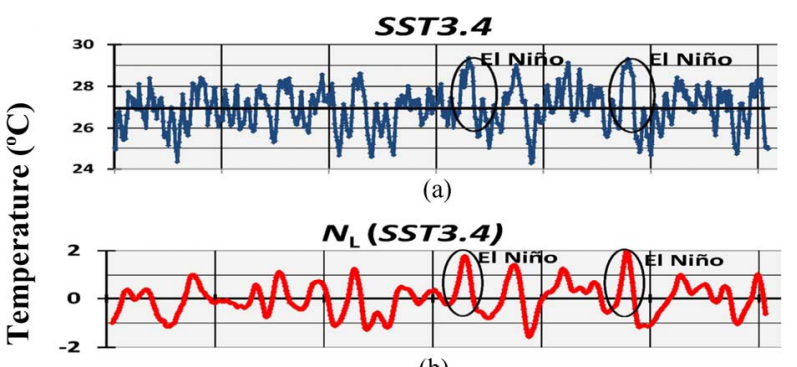

(b)

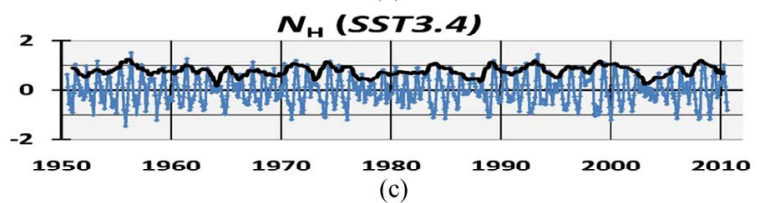

(c)

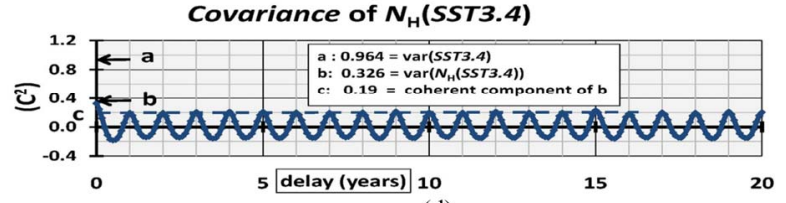

(d)

Figure 1. Plots based upon SST3.4: (a) SST3.4, Black line is the average; (b) $N_{\mathrm{L}}(S S T 3.4)$, the low frequency component of SST3.4; Two EI Niños are indicated; (c) $N_{\mathrm{H}}$ (SST3.4) (blue), the high frequency component of $S S T 3.4$ and its amplitude $A_{\mathrm{H}}(S S T 3.4)$ (black); (d) Covariance of $N_{\mathrm{H}}(S S T 3.4)$ vs. lag $\tau$. The variance of $0.326\left(C^{2}\right)$ at $\tau=0$ is indicated.

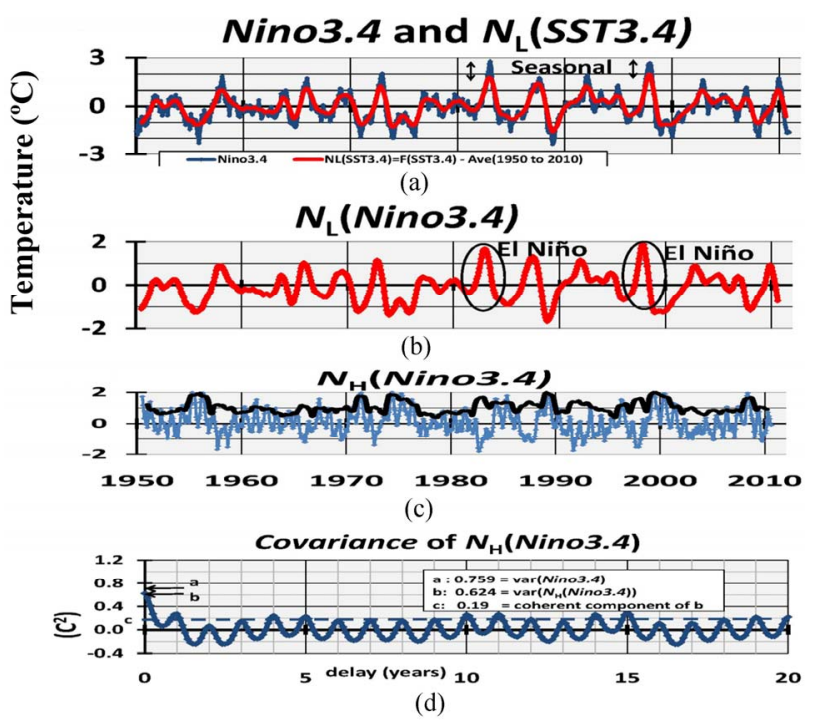

Figure 2. Plots based upon index Nino3.4: (a) Nino3.4 (blue) and Index $N_{\mathrm{L}}$ (SST3.4) (red); (b) $N_{\mathrm{L}}$ (Nino3.4), the low frequency component of Nino3.4; (c) $N_{\mathrm{H}}$ (Nino3.4) (blue), the high frequency component of Nino3.4 and its amplitude $A_{\mathrm{H}}$ (Nino3.4) (black); (d) Covariance of $N_{\mathrm{H}}$ (nino3.4) vs. lag $\tau$. The variance of $0.624\left(C^{2}\right)$ at $\tau=0$ is indicated.

$$
\begin{aligned}
& \mathcal{F}=12 \text {-month symmetric moving average } \\
& \text { "box" digital filter. }
\end{aligned}
$$

This filter is a low pass filter that allows frequencies 
lower than $(1 / 12)$ month $^{-1}$ to pass with only slight attenuation. This filter has an additional important property that is not generally recognized. The Fourier transform of $\mathcal{F}$ is $H_{12}(f)=\sin (\pi 12 f) / \sin (\pi f)$, which has zeros at multiples of the frequency $f=(1 / 12)$ month $^{-1}$ [5]. Thus, signals whose frequencies are exactly $f=(1 / 12)$ month $^{-1}$ and its harmonics are removed. This second property is highly desirable in reducing an unwanted seasonal signal that contains an annual component and its harmonics. One frequently sees the use of $k$-month filters where $k$ has values $3,5,7,9,11,12,13$ in attempts to reduce the seasonal signal; the $k=12$ filter is obviously best for removal of such a signal. Because the center of this filter is between time series data points, one has to place the center of the filter one half interval before or after the reference data point. Here, the choice is "after". The 12month filter is then described as "6-1-5", where "1" is the reference. There is a loss of six data points at the beginning of the time series and five at end of the timeseries.

\subsection{Climate Indices}

The filter $\mathcal{F}$ is applied to a time series $G$ to create a "low frequency" index $N_{\mathrm{L}}$,

$$
N_{\mathrm{L}}(G)=\mathscr{F}(G)-\text { average } \mathscr{F}(G),
$$

where the average is over a range of $G$ (1981-2010 in this paper) and a "high frequency" index

$$
N_{\mathrm{H}}(G)=G-\mathcal{F}(G) .
$$

Equation (2) been applied to SST3.4 data by Douglass [1] to define a new El Niño/La Niña index $N_{L}(S S T 3.4)$. This is shown in Figure 1(b). Application of Equation (3) to $S S T 3.4$ creates $N_{\mathrm{H}}(S S T 3.4)$, which contains the seasonal signal. $N_{\mathrm{H}}(S S T 3.4)$ is plotted in Figure 1(c), which shows a strong periodicity of one year. The amplitude of $N_{\mathrm{H}}$ is defined as

$$
A_{\mathrm{H}}=2^{1 / 2} R M S\left(N_{\mathrm{H}}\right),
$$

where $R M S$ is the square root of the symmetric 12month mean square of $N_{\mathrm{H}} . A_{\mathrm{H}}$ of $N_{L}(S S T 3.4)$ is shown in Figure 1(c) (black).

The variously defined quantities are listed in Table 1.

\subsection{Covariance}

The covariance (covar) of a signal $x(t)$ is defined as

$$
\operatorname{covar}(\tau)=\frac{1}{K-1} \sum_{1}^{K} x(t) x(t-\tau)=\operatorname{var}(x) \rho_{x}(\tau) ;
$$

\begin{tabular}{|c|c|c|}
\hline \multicolumn{3}{|c|}{ Definitions } \\
\hline$G$ & $\begin{array}{l}\text { A geophysical time series that contains a signal } N \text { of interest plus a } \\
\text { seasonal signal of known period. }\end{array}$ & $\begin{array}{l}\text { SST3.4 is an example which contains the E1 Niño/La } \\
\text { Niña phenomenon plus a seasonal signal of period } \\
\text { 12-months. }\end{array}$ \\
\hline Filter $\mathcal{F}$ & n-month symmetric moving average "box" digital filter. $\mathrm{n}=12$ for $S S T 3.4$ & Used to create the two indices in the next two rows. \\
\hline$N_{\mathrm{L}}(G)$ & $N_{\mathrm{L}}=\mathscr{F}(G)-$ average $\mathcal{F}(G)$ & This is the "low frequency" component of $G$. \\
\hline$N_{\mathrm{H}}(G)$ & $N_{\mathrm{H}}=T-\mathcal{F}(G)$ & This is the "high frequency" component of $G$. \\
\hline$A_{\mathrm{H}}(G)$ & $A_{\mathrm{H}}=2^{1 / 2} R M S\left(N_{\mathrm{H}}\right)$ & Amplitude of $N_{\mathrm{H}}$. \\
\hline Region 3.4 & Area in central Equatorial Pacific [5S to $5 \mathrm{~N} ; 120 \mathrm{~W}$ to $170 \mathrm{~W}]$ & $\begin{array}{l}\text { A primary reference area for El Niño/La Niña } \\
\text { phenomenon. }\end{array}$ \\
\hline SST3.4 & $\begin{array}{l}\text { Monthly values of the average Sea Surface Temperature (SST) over } \\
\text { region 3.4. }\end{array}$ & $\begin{array}{l}\text { Ranges between } 24^{\circ} \mathrm{C} \text { and } 30^{\circ} \mathrm{C} \text {. Consists of the } \mathrm{El} \\
\text { Niño/La Niña phenomenon plus a seasonal effect of } \\
\text { 1-year period. }\end{array}$ \\
\hline Climatology & $\begin{array}{l}\text { For monthly data the Climatology }(C) \text { is a set of } 12 \text { constant numbers that } \\
\text { are an average of } G \text { for each month over a fixed period (usually } 30 \text { years). }\end{array}$ & $\begin{array}{l}\text { Used in the construction of the index of anomalies such } \\
\text { as Nino3.4. }\end{array}$ \\
\hline Nino3.4 & The commonly used index of anomalies that is defined as SST3.4 minus C & $\begin{array}{l}\text { It is stated that the seasonal effect has been "removed". } \\
\text { The text explains that this is not true. }\end{array}$ \\
\hline \multicolumn{3}{|c|}{ Chaotic Properties of a Dynamic System } \\
\hline & Lyapunov exponents & Comments \\
\hline & $\begin{array}{l}\text { At least one exponent }>0 \text {. } \\
\text { Sum of exponents }<0 \text {. }\end{array}$ & $\begin{array}{l}\text { means dissipative dynamics. } \\
\text { signal is "well-defined". }\end{array}$ \\
\hline & $\begin{array}{l}\text { At least one exponent }>0 \text {. } \\
\text { Sum of exponents }>0\end{array}$ & e signal is "ill-defined". \\
\hline
\end{tabular}

where $t$ is a time lag; the summation is over the equally spaced values of $x ; \operatorname{var}(x)$ is the usual statistical variance

Table 1. Definitions of various quantities. 
and $r(t)$ is the commonly used delayed Pearson autocorrelation function. When $t=0, \operatorname{covar}(0)=\operatorname{var}(x)$. For later reference it is noted that if $x$ is a random variable, there is very little covariance after $t=0$.

\section{Analysis}

The average, variance and trend of various time series from SST3.4 were calculated and are listed in Table 2(a). Also listed are the magnitudes of the El Niños of 19821983 and 1997-1998 according to several different indices.

Index $N_{\mathrm{L}}$ was computed for SST3.4 and is shown in Figure 1(b). The seasonal signal $N_{\mathrm{H}}(S S T 3.4)$ and its amplitude $A_{\mathrm{H}}(S S T 3.4)$ are shown in Figure 1(c). Of particular interest is the covariance of $N_{\mathrm{H}}($ SST3.4) vs. lag $t$ shown in Figure 1(d). For large lags, the covariance of $N_{\mathrm{H}}$ (SST3.4) shows periodic behavior at a period of 1 year of amplitude $0.20 \mathrm{C}^{2}$ which indicates a sustained oscillation almost certainly of solar origin. Computation of the average value for each month of $N_{\mathrm{H}}$ (SST3.4) shows that the maximum $(0.75 \mathrm{C})$ occurs during Apr/ May and that a broad minimum $(-0.43 \mathrm{C})$ occurs during Dec/Jan. The covariance of $N_{\mathrm{H}}($ SST3.4) at $t=0$ is the variance whose value is $0.326 \mathrm{C}^{2}$. Since the variance of

Table 2. (a) Statistical and other properties of SST3.4 and Niño3.4; (b) Variances.

(a)

\begin{tabular}{cccccccc}
\hline \multicolumn{7}{c}{ Statistical and other properties } \\
\hline \multicolumn{7}{c}{$S S T 3.4$} & \multicolumn{5}{c}{ Nino3.4 } \\
\hline & SST3.4 & $N_{\mathrm{L}}$ & $N_{\mathrm{H}}$ & Nino3.4 & $N_{\mathrm{L}}$ & $N_{\mathrm{H}}$ \\
$\begin{array}{c}\text { Average (C) } \\
(1950-2010)\end{array}$ & 26.942 & 0.000 & 0.003 & -0.096 & -0.081 & 0.083 \\
$\begin{array}{c}\text { Variance (C2) } \\
(1950-2010)\end{array}$ & 0.964 & $\begin{array}{c}0.458 \\
\text { (Note A) }\end{array}$ & 0.326 & 0.759 & $\begin{array}{c}0.458 \\
\text { (Note A) }\end{array}$ & 0.624 \\
$\begin{array}{c}\text { Slope (C/decade) } \\
(1950-2010)\end{array}$ & 0.043 & 0.045 & 0.003 & 0.044 & 0.045 & -0.042 \\
$\begin{array}{c}\text { Amplitude El Niño } \\
\text { of 1982-1983 (C) }\end{array}$ & 1.78 & & & 2.79 & \\
$\begin{array}{c}\text { Amplitude El Niño } \\
\text { of 1997-1998 (C) }\end{array}$ & 1.98 & & & 2.69 & \\
\hline
\end{tabular}

Note A. These two values are very close. The 12 climatology values used to calculate Nino3.4 are: 26.6, 26.7, 27.2, 27.8, 27.9, 27.7, 27.2 26.8, 26.7, 26.7, 26.7, and $26.6(\mathrm{C})$.

(b)

\begin{tabular}{|c|c|c|c|c|c|}
\hline \multicolumn{6}{|c|}{ Comparison of variances $\left(C^{2}\right)$} \\
\hline & & SST3.4 & & Nino3.4 & \\
\hline Parent & 0.964 & & 0.759 & & \\
\hline$N_{\mathrm{H}}$ & & 0.326 & & 0.624 & \\
\hline$N_{\mathrm{H} \_ \text {random }}{ }^{\mathbf{a}}$ & & $\approx 0.13$ & & & $\approx 0.43$ \\
\hline$N_{\mathrm{H}}$ coherent $^{\mathrm{a}}$ & & $\approx 0.20$ & & & $\approx 0.19$ \\
\hline
\end{tabular}

Note a. The random component of the variance is the amount of the abrupt drop in covar( $(0)$. The rest of covar(0) is the coherent component.

the coherent signal is $0.20 \mathrm{C}^{2}$, then the difference from $0.326 \mathrm{C}^{2}$ of $\sim 0.19 \mathrm{C}^{2}$ can be inferred to be due to random processes. The variance of the parent signal, SST3.4 of $0.964 \mathrm{C}^{2}$ is also indicated on the plot. Thus, SST3.4 contains a seasonal signal whose variance is about $1 / 3$ of that of SST3.4. The seasonal signal consists of two components whose variances are: a coherent signal at a period of 1 year (variance $=0.20 \mathrm{C}^{2}$ ) and a random signal (variance $=0.13 \mathrm{C}^{2}$ ).

The same calculations were carried out on index Nino3.4 and are shown in Figures 2(a-d). What is the effect of using the climatology to produce index Nino3.4? The variance of Nino3.4 is $0.749 \mathrm{C}^{2}$ which is less than the $0.964 \mathrm{C}^{2}$ value of SST3.4. However, the variance of the seasonal signal, $N_{\mathrm{H}}\left(\right.$ Nino3.4), is $0.624 \mathrm{C}^{2}$. Thus, not only is the variance of the seasonal signal not reduced by the climatology method but it is now nearly twice in magnitude! This "not removed" seasonal signal consists of two components whose variances are: a coherent signal at a period of 1 year (variance $=0.19 \mathrm{C}^{2}$ ) and a random signal (variance $=0.43 \mathrm{C}^{2}$ ). The climatology method has increased the random component of the seasonal signal while keeping the coherent component nearly the same. In sum, the climatology method does not remove the seasonal signal from SST3.4.

\section{Discussion}

\subsection{Separation of the Seasonal Signal}

One of the main conclusions of this study is that the "season free" index Nino3.4 contains a substantial component of a seasonal signal. Thus the climatology method fails. Furthermore, no set of climatology values will remove the seasonal signal from SST3.4. This is because the climatology is a function consisting of a constant plus a periodic function with monthly amplitudes that are the same for each year while the seasonal signal $N_{\mathrm{H}}$ calculated from the SST3.4 data (See Figure 1(c)) is also a periodic function the amplitude is not a constant. Thus, the climatology method subtracts an incorrect periodic signal from the parent SST3.4 signal and has produced a different seasonal signal that is larger than it was before.

Somewhat miraculously, the filter $\mathcal{F}$ applied to Nino3.4 will remove both the incorrect seasonal function introduced by the climatology method and the original seasonal signal. So $N_{\mathrm{L}}\left(\right.$ Nino3.4) $\approx N_{\mathrm{L}}$ (SST3.4), which agrees with the observation of Douglass [1].

It is important to know the extent to which Nino3.4 is "contaminated" by a seasonal signal because this index is widely used. For example, the United States National 
Oceanic and Atmospheric Administation and 26 nations of the world officially use Nino3.4 for monitoring and predicting El Niño and La Niña conditions [6]. This study shows that the Nino3.4 estimates of the magnitude of El Niños are larger than estimates from $N_{\mathrm{L}}$ (SST3.4) in agreement with Douglass [1]. For example, the magnitudes for the 1997-1998 El Niño listed in Table 2(a) are $2.69^{\circ} \mathrm{C}$ and $1.98^{\circ} \mathrm{C}$ respectively. More importantly, the relative magnitudes are also different. Index $N_{\mathrm{L}}($ SST3.4) shows that the magnitude of the El Niño of 1997-1998 is larger than the magnitude of the El Niño of 1983-1984 in agreement with the ordering by the National Climate Data Center [7]. The relative magnitudes from index Nino3.4 shows the opposite ordering.

\subsection{A New Measure for Determining the Presence of a Seasonal Component.}

In a dynamic system of $d$ degrees of freedom perturbations grow or decay as $e^{\lambda_{L Y P} t}$, where $t$ is the time and $l_{L Y P}$ is the Lyapunov exponent—one for each $d$ [8]. Positive exponents indicate growth while negative exponents indicate decay. The volume in $d$-space of the perturbation grows or decays as the sum $S$ of the exponents. For systems of finite energy with dissipation the volume must decay, which requires that $S$ be negative. If $S$ is positive, then the finite energy condition or the dissipation condition on the system are not satisfied.

The Lyapunov exponents can be calculated using the methods of chaos theory developed by the nonlinear dynamics community. Abarbanel's book [8] is unique in that it is a "tool kit" on how the chaotic properties can be readily determined from the study of an appropriate scalar time series from that system. A set of programs to calculate $d$ and the $l \mathrm{~s}$ is available from Randle Inc. [9] or from Abarbanel. An outline of the steps from the "tool kit" is now given.

One starts with the premise that the physical dynamical system is nonlinear and chaotic. If the system is not chaotic, then that will be known if none of the Lyapunov exponents are positive. The first step is selecting a scalar time series from the physical dynamical system (e.g. SST3.4). If the time series contains "noise" then it must be separated by some method such as with a moving average filter. For the case of the SST3.4 the "noise" is the seasonal signal. Next, one determines $d$ and the Lyapunov exponents. For the index time series considered in this paper, $d=3$. The properties of the underlying dynamic system are determined from the set of exponents.

1) By definition, the system is chaotic if one of the $l$ 's is positive.

2) Since physical systems of finite energy are dissipative, the sum $S$ of the exponents must be negative. Such a system will be called well-defined.

3) A system having time series with a positive $S$ will be called ill-defined. Empirically, it is found that this case corresponds to the presence of a seasonal signal.

It is postulated that:

If $S$ is negative, the index is season free.

If $S$ is positive, the index contains a seasonal signal.

The Abarbanel chaos analysis was applied to the SST3.4, $N_{\mathrm{L}}, N_{\mathrm{H}}$, Niño3.4, and $N_{\mathrm{L}}($ Niño3.4) time series. The first outcome is that for all time series the dimension $d$ is 3. The Lyapunov exponents and their sum $S$ are listed in Table 3. In addition, at least one of the Lyapunov exponents is positive, thus verifying the presumption that these time series come from processes that are nonlinear and chaotic. This will be explored in a later publication. The issue of the sum $S$ of exponents is illustrated in Figure 3. The $S$ for the fundamental time series SST3.4 is 0.385 . This is positive and thus SST3.4 is ill-defined (by hypothesis because of the seasonal signal). The new El Niño/La Niña index, $N_{\mathrm{L}}$ (SST3.4) has $S=$ -0.119 , which is negative and thus well-defined. For Nino3.4 (the index derived by subtracting the climatology from SST3.4) the $S$ is +0.198 which has been somewhat reduced from that of SST3.4 but is still positive and thus

Table 3. Lyapunov exponents.

\begin{tabular}{ccccccc}
\hline \multicolumn{7}{c}{ Lyapunov Exponents $^{\left(\text {months }^{-1} \text { ) }\right.}$} \\
\hline & & SST3.4 & $\boldsymbol{N}_{\mathbf{L}}$ & $\boldsymbol{N}_{\mathbf{H}}$ & Nino3.4 & $\mathcal{F}($ Nino3.4) \\
Lyapunov & $\lambda 1$ & 0.587 & 0.243 & 0.781 & 0.560 & 0.315 \\
$d_{E}=d_{L}=3$ & $\lambda 2$ & 0.220 & $0.031[\mathrm{zero}]$ & 0.356 & 0.241 & $0.051[\mathrm{zero}]$ \\
& $\lambda 3$ & -0.422 & -0.393 & -0.327 & -0.503 & -0.446 \\
Note & Sum & 0.385 & -0.119 & 0.814 & 0.198 & -0.080 \\
& & $\mathrm{~B}$ & $\mathrm{~A}$ & $\mathrm{~B}$ & $\mathrm{~B}$ & $\mathrm{~A}$ \\
\hline
\end{tabular}

Note A: Sum of exponents $<0$. The time series is well-defined. Sum of exponents $<0$ means dissipative. If, in addition, one of the exponents $=0$, then the dynamics can be described by ordinary differential equations. Note B: Sum of exponents $>0$. The time series is ill-defined due to a lack of separation of the seasonal effect. 


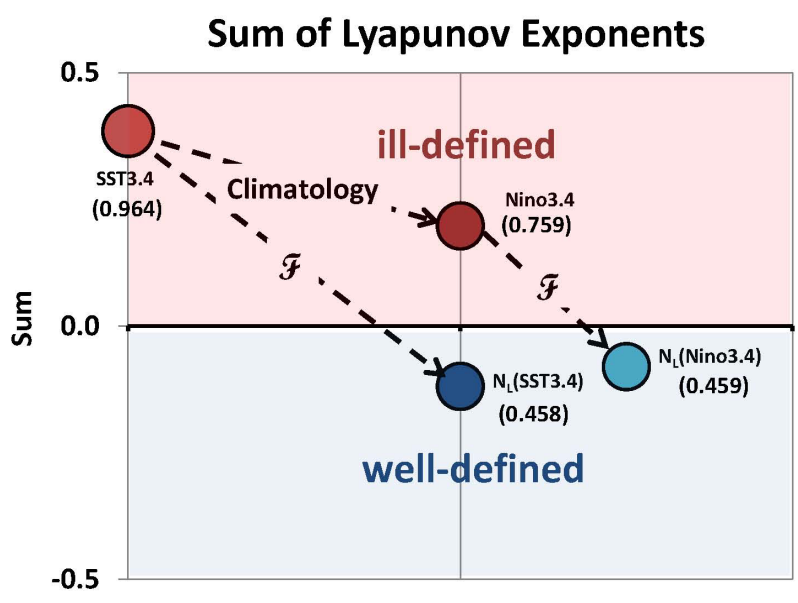

Figure 3: Schematic showing the sum of Lyapunov exponents of various indices derived from Region3.4. From the chaos test that the sum of the Lyapunov exponents must be negative one finds that only $N_{\mathrm{L}}\left(\right.$ SST3.4) and $N_{\mathrm{L}}($ Nino3.4) are well-defined. Numbers in parentheses are the variances from Table 1. Arrows labeled with $F$ indicates a process that used the 12-month digital filter.

ill-defined; by hypothesis, the seasonal signal has not been completely separated. However, $N_{\mathrm{L}}$ (Nino3.4) (applying filter $\mathcal{F}$ to Nino3.4) has an $S$ of -0.080 in- dicating that this index is well-defined.

\section{Summary and Conclusions}

The commonly used climatology method of subtracting constant seasonal values of SST3.4 to produce the widely used El Niño/La Niña index Nino3.4 is shown to fail because this index still contains a substantial seasonal signal. Furthermore, no set of constant seasonal values will remove the seasonal signal because the seasonal values are not constant.

A different scheme is given that does not use the climatology method. Using a moving average filter $\mathcal{F}$ one can create a signal $N_{\mathrm{L}}$ that contains the low frequency effect of interest, such as the El Niño/La Niña phenome- non, and a high frequency signal $N_{\mathrm{H}}$ that contains the seasonal signal. Various tests including one based upon chaos properties show that $N_{\mathrm{L}}$ is "seasonal free".

These results are applicable to other geophysical measurements.

\section{Acknowledgements}

Many helpful discussions were held with H. D. I. Arbarbanel and R. S. Knox.

\section{References}

[1] D. H. Douglass. "El Niño Southern Oscillation: Magnitudes and Asymmetry," Journal of Geophysical Research, Vol. 115, No. D15, pp. 1-6. doi:10.1029/2009JD013508

[2] A. V. Federov and S. G. Philander, "Is El Nino Sporadic or Cyclic?" Annual Review of Earth and Planetary Sciences, Vol. 31, No. 31, pp. 579-594, doi:10.1146/annurev.earth.31.100901.141255.2003

[3] NOAA/CPC. SST Data and Indices at 2009 http://www.cpc.ncep.noaa.gov/data/indices/

[4] A. C. Barnston, M. Chelliah and S. Goldenberg, "Documentation of a Highly ENSO Related SST Region in the Equatorial Pacific," Atmosphere-Ocean, Vol. 35, No. 3, 1997, pp. 367-383. doi:10.1080/07055900.1997.9649597

[5] S. W. Smith, "The Scientists and Engineer's Guide to Signal Processing," California Technical Publishing, San Diego, 1997.

[6] NOAA, World Meteorological Organization Adopts Consensus El Niño and La Niña index and Definitions, 1995. http://www.noaanews.noaa.gov/stories2005/s2428.htm26,

[7] NCDC, The top $10 \mathrm{El}$ Nino Events of the $20^{\text {th }}$ Century, 2011.

http://www.ncdc.noaa.gov/oa/climate/research/1998/enso /10elnino.html

[8] H. D. I. Abarbanel, "Analysis of Observed Chaotic Data," Springer-Verlag, Berlin, 1995.

[9] Randle Inc. cspW, Tools for Dynamics, Applied Nonlinear Sciences, LLC, Randle Inc., Great Falls, 1996. 